\title{
APOE-by-sex interactions on brain structure and metabolism in healthy elderly controls
}

\author{
Frederic Sampedro ${ }^{1,2,3, *}$, Eduard Vilaplana ${ }^{1,2, *}$, Mony J de Leon ${ }^{4}$, Daniel Alcolea ${ }^{1,2}$, \\ Jordi Pegueroles ${ }^{1,2}$, Victor Montal ${ }^{1,2}$, María Carmona-Iragui ${ }^{1,2}$, Isabel Sala ${ }^{1,2}$, \\ María-Belén Sánchez-Saudinos ${ }^{1,2}$, Sofía Antón-Aguirre ${ }^{1,2}$, Estrella Morenas- \\ Rodríguez $^{1,2}$, Valle Camacho ${ }^{3}$, Carles Falcón ${ }^{5,7}$, Javier Pavía ${ }^{6,7}$, Domènec $\operatorname{Ros}^{5,7}$, \\ Jordi Clarimón ${ }^{1,2}$, Rafael Blesa ${ }^{1,2}$, Alberto Lleó ${ }^{1,2}$, Juan Fortea ${ }^{1,2}$ for the Alzheimer's \\ Disease Neuroimaging Initiative** \\ ${ }^{1}$ Memory Unit, Department of Neurology, Hospital de la Santa Creu i Sant Pau- Biomedical Research Institute Sant \\ Pau- Universitat Autònoma de Barcelona, Barcelona, Spain \\ ${ }^{2}$ Centro de Investigación Biomédica en Red de Enfermedades Neurodegenerativas. CIBERNED, Madrid, Spain \\ ${ }^{3}$ Nuclear Medicine Department, Hospital de la Santa Creu i Sant Pau- Biomedical Research Institute Sant Pau- Universitat \\ Autònoma de Barcelona, Barcelona, Spain \\ ${ }^{4}$ New York University School of Medicine, New York, NY, USA \\ ${ }^{5}$ Unitat de Biofísica i Bioenginyeria, Departament de Ciències Fisiològiques I, Facultat de Medicina, Universitat de Barcelona \\ - IDIBAPS, Barcelona, Spain \\ ${ }^{6}$ Nuclear Medicine Department. Hospital Clínic de Barcelona, Barcelona, Spain \\ ${ }^{7}$ Biomedical Research Networking Center in Bioengineering, Biomaterials and Nanomedicine - CIBER-BBN, Barcelona, Spain \\ *These authors have contributed equally to this work \\ *** Data used in preparation of this article were obtained from the Alzheimer's Disease Neuroimaging Initiative (ADNI) \\ database (adni.loni.usc.edu). As such, the investigators within the ADNI contributed to the design and implementation of \\ ADNI and/or provided data but did not participate in analysis or writing of this report. A complete listing of ADNI investigators \\ can be found at: http://adni.loni.usc.edu/wp-content/uploads/how_to_apply/ADNI_Acknowledgement_List.pdf.
}

Correspondence to:

Juan Fortea, e-mail: jfortea@santpau.cat

Keywords: Gerotarget, Alzheimer's disease, aging, APOE, MRI, PET-FDG

Received: June 29, 2015

Accepted: August 28, 2015

Published: September 10, 2015

\section{ABSTRACT}

Background: The APOE effect on Alzheimer Disease (AD) risk is stronger in women than in men but its mechanisms have not been established. We assessed the APOE-by-sex interaction on core CSF biomarkers, brain metabolism and structure in healthy elderly control individuals (HC).

Methods: Cross-sectional study. HC from the Alzheimer's Disease Neuroimaging Initiative with available CSF $(n=274)$ and/or 3T-MRI $(n=168)$ and/or a FDGPET analyses $(n=328)$ were selected. CSF amyloid- $B_{1-42}\left(A \beta_{1-42}\right)$, total-tau (t-tau) and phospho-tau $\left(\mathrm{p}-\mathrm{tau}_{181 \mathrm{p}}\right.$ ) levels were measured by Luminex assays. We analyzed the APOE-by-sex interaction on the CSF biomarkers in an analysis of covariance (ANCOVA). FDG uptake was analyzed by SPM8 and cortical thickness (CTh) was measured by FreeSurfer. FDG and CTh difference maps were derived from interaction and group analyses.

Results: APOE4 carriers had lower CSF $A \beta_{1-42}$ and higher CSF p-tau ${ }_{181 \mathrm{p}}$ values than non-carriers, but there was no APOE-by-sex interaction on CSF biomarkers. The $A P O E-b y-s e x$ interaction on brain metabolism and brain structure was significant. Sex stratification showed that female APOE4 carriers presented widespread brain hypometabolism and cortical thinning compared to female non-carriers whereas male 


\section{APOE4 carriers showed only a small cluster of hypometabolism and regions of cortical thickening compared to male non-carriers.}

\section{Conclusions: The impact of APOE4 on brain metabolism and structure is modified by sex. Female APOE4 carriers show greater hypometabolism and atrophy than male carriers. This $A P O E-b y-s e x$ interaction should be considered in clinical trials in preclinical AD where APOE4 status is a selection criterion.}

\section{INTRODUCTION}

The apolipoprotein $\mathrm{E}(A P O E)$ genotype is the strongest genetic risk factor for Alzheimer's disease (AD) [1]. It has three isoforms, $\varepsilon 2, \varepsilon 3$ and $\varepsilon 4$. The $A P O E$ $\varepsilon 4$ allele (APOE4) increases the risk for $\mathrm{AD}$ [2]. The effect of the $A P O E 4$ allele on AD biomarkers in healthy controls (HC) has been widely studied [3], [4]. APOE4 carriers have consistently lower cerebrospinal fluid (CSF) ß-amyloid 1-42 $\left(\mathrm{A} \beta_{1-42}\right)$ levels than non-carriers, but the differences in tau levels are more controversial [5]-[7]. Most, [8]-[10] but not all [18F]-fluorodeoxyglucose (FDG) PET studies [11]-[13] have shown hypometabolism in $\mathrm{AD}$-related regions in APOE4 carriers in late-middle age [8] and even earlier [10]. A gene-dosage effect on the hypometabolism has also been reported [9]. The relationship between the $A P O E$ genotype and brain structure is more controversial. Many cross-sectional studies have reported cortical thinning or hippocampal atrophy, [3], [4], [14] while several others have found no relationship [15] and two have reported increased gray matter in relation to the APOE4 allele [16], [17].

Several factors might account for the conflicting results. First, the age-range differences between studies are critical because distinct effects of $A P O E$ across the lifespan have been described [18]. Not all brain changes associated with the $A P O E$ genotype reflect incipient AD. $A P O E$ has been implicated in normal human brain development [19]. Second, there are amyloid dependent [20] and independent [21] mechanisms underlying the $A P O E$ influences on $\mathrm{AD}$ risk. However, most studies assessing the role of $A P O E$ on brain structure and metabolism do not assess AD pathophysiological biomarkers to disentangle these mechanisms. Third, APOE4 is likely to interact with other pathological factors, complicating the isolation of a unique genetic effect [4]. And fourth, some of the inconsistent imaging and biochemical findings related to $A P O E$ in $\mathrm{HC}$ might result from neglecting a possible $A P O E$-by-sex interaction [6]. Most studies to date have included sex as a covariate in the analyses but they did not explicitly test for an $A P O E$-by-sex interaction.

The finding that the $A P O E$ effect on $\mathrm{AD}$ risk is stronger in women than in men was reported in early studies, [22], [23] confirmed in meta-analyses, [23], [24] and in a recent longitudinal study [6]. However, only two studies have assessed $A P O E$-by-sex interactions on AD biomarkers. Altmann et al found a significant interaction for tau in mild cognitive impairment patients [6]. Damoiseaux et al reported a significant $A P O E$-by-sex interaction for CSF tau levels and default mode network abnormalities in healthy controls [25].

The interaction between APOE4 and sex on brain structure and metabolism has not been established. This interaction could affect the design and interpretation of prevention trials in preclinical $\mathrm{AD}$ in which APOE is a selection criterion (i.e. the Alzheimer's Prevention Initiative $A P O E 4$ Trial, NIH project number 1UF1AG046150-01). The aim of the present study was to examine the interactions between $A P O E 4$ and sex on brain metabolism and structure, based on the hypothesis that the APOE4 allele exerts a differential adverse effect on brain metabolism and structure depending on sex.

\section{RESULTS}

Demographic and clinical of the participants in the CSF, FDG and MRI subsets are summarized separately in the Table 1. CSF was available in $274 \mathrm{HC}$ individuals, 328 had an FDG PET, 225 had a 3T MRI, and 137 subjects had all three biomarkers. There were no significant differences between the MRI, PET and CSF subsets in age, sex, APOE status, MMSE or CSF biomarkers. There were no significant differences in age, APOE status, MMSE or CSF biomarkers between males and females in all three subsets. In the FDG and CSF subsets, males had higher years of education than females $(p<0.001)$, but in the MRI subset this difference did not reach significance.

APOE4 carriers had lower CSF $A \beta_{1-42}$ values than non-carriers in all three subsets $(p<0.001)$. APOE4 carriers had higher CSF p-tau ${ }_{181 \mathrm{p}}$ values in the three subsets, but these only reached significance in the FDG and CSF subset which had larger sample sizes $(p<0.001$ and $p=0.004$ respectively). APOE 4 carriers had higher CSF t-tau values in the three subsets, but these only reached significance in the CSF subset $(p<0.05)$. There were no significant differences in MMSE scores or education between APOE4 carriers compared to non-carriers in any of the subsets. There were no significant differences between males and females in CSF biomarkers. Neither was there an $A P O E$-by-sex interaction on CSF A $\beta_{1-42 .}$ CSF $\mathrm{t}$-tau or CSF $\mathrm{p}$-tau ${ }_{181 \mathrm{p}}$ values in the analysis of covariance (ANCOVA) analyses. 
Table 1: Demographic, cerebrospinal fluid and clinical data in the CSF, FDG-PET and MRI Alzheimer's Disease Neuroimage Initiative subsets.

\begin{tabular}{|c|c|c|c|c|}
\hline \multirow{2}{*}{\multicolumn{2}{|c|}{ APOE4 N $\%$}} & MRI $(N=168)$ & FDG-PET $(N=328)$ & $\operatorname{CSF}(N=274)$ \\
\hline & & $50(29.76 \%)$ & $87(26.5 \%)$ & $71(25.9 \%)$ \\
\hline \multicolumn{2}{|l|}{ AGE } & $73.4(6.02)$ & $74.5(5.57)$ & $74.4(5.97)$ \\
\hline \multicolumn{2}{|c|}{ SEX (\% Females) } & $53.6 \%$ & $49.4 \%$ & $50.4 \%$ \\
\hline \multicolumn{2}{|l|}{ MMSE } & $29.1(1.07)$ & $29.0(1.24)$ & $29.1(1.15)$ \\
\hline \multicolumn{2}{|c|}{ YEARS OF EDUCATION } & $16.6(2.55)$ & $16.3(2.77)$ & $16.3(2.69)$ \\
\hline \multirow[t]{3}{*}{$\mathbf{A} \mathbf{B}_{1-42} * * *$} & TOTAL & $200.7(49.92)$ & $201.4(52.46)$ & $200.6(52.51)$ \\
\hline & APOE4- & $211.3 *(46.32)$ & $213.5 *(46.87)$ & $212.1 *(47.81)$ \\
\hline & APOE4+ & $175.4 *(49.58)$ & $165.2 *(51.85)$ & $167.9 *(51.87)$ \\
\hline \multirow[t]{3}{*}{ p-tau ${ }_{\mathrm{p} 181} * * *$} & TOTAL & $32.4(16.41)$ & $30.78(18.14)$ & $30.48(17.97)$ \\
\hline & APOE4- & $31.3(16.68)$ & $28.3 *(15.31)$ & $28.2 *(15.23)$ \\
\hline & APOE4+ & $35.0(15.62)$ & $38.1 *(23.38)$ & $36.9 *(23.10)$ \\
\hline \multirow[t]{3}{*}{ t-tau*** } & TOTAL & $66.0(31.88)$ & $68.9(34.57)$ & $68.4(32.12)$ \\
\hline & APOE4- & $65.1(32.60)$ & $67.0(34.84)$ & $66.0 * *(30.29)$ \\
\hline & APOE4+ & $68.2(30.34)$ & $74.5(33.41)$ & $75.1 * *(36.22)$ \\
\hline
\end{tabular}

APOE4+ = apolipoprotein E $\varepsilon 4$ allele carrier, $\mathrm{APOE}-=$ apolipoprotein $\mathrm{E} \varepsilon 4$ allele non-carrier

Values are expressed as mean (standard deviation) unless specified.

*equals $p<0.001$ and

** equals $p<0.05$ for the $A P O E 4$ carriers vs non-carriers comparison within each subset. Note that 137 subjects were included in the three subsets.

${ }^{* * *}$ CSF data only available in 146 subjects in the MRI subset and 242 subjects in the PET subset.

\section{$A P O E$-by-sex interaction on brain metabolism}

Fig. 1A presents this FDG voxel-wise interaction analysis across the cerebral hemispheres, showing voxels with an $A P O E$-by-sex interaction, covaried by age and years of education $(p<0.005, k=50)$. Two clusters emerged, one located mainly in the anterior cingulate region and the other in the temporal region. To analyze the directionality, we isolated the temporal cluster, averaged the FDG uptake, and plotted it in box and whisker plots (Fig. 1B). As shown, this interaction was driven by the decreased metabolism in female APOE4 carriers and the increased metabolism in male $A P O E 4$ carriers. The main and interactive effects of APOE4 status and sex on brain metabolism in the ANCOVA analysis were significant in the model (interaction term between APOE4 status and sex: $\beta$-coefficient $=0.069$, standard error $[\mathrm{SE}]=0.021, p=0.001$; main effect of $A P O E 4$ status: $\beta$-coefficient $=-0.037, \mathrm{SE}=0.016, p=0.019$; main effect of sex: $\beta$-coefficient $=-0.041, \mathrm{SE}=0.018, p=0.026$ ). Similar results were found for the anterior cingulate cluster (not shown).

Fig. 2 shows the sex stratified APOE4 group analyses for FDG, covaried by age and years of education. Female APOE4 carriers showed widespread clusters of decreased metabolism $(p<0.005)$ across the whole cerebral cortex in both hemispheres with respect to APOE4 non-carriers (Fig. 2A). Male APOE4 carriers showed an isolated cluster of decreased metabolism $(p<0.005)$ in the precuneus with respect to non-carriers (Fig. 2B).

To examine the impact of CSF biomarkers in the $A P O E$-by-sex interaction on brain metabolism, we included CSF $A \beta_{1-42}$ and CSF p-tau ${ }_{181 \mathrm{p}}$ as covariates in the analyses. The inclusion of the CSF biomarkers did not significantly alter the results of the $A P O E$-by-sex interaction analysis (not shown) nor the female $A P O E 4$ carriers vs non-carriers comparison (Fig. 3A1-3A3). In the male APOE4 carriers vs non-carriers comparison two clusters of increased metabolism emerged in APOE4 carriers with respect to male non-carriers in prefrontal regions and a cluster in the medial temporal region when CSF $A \beta_{1-42}$ levels or both $A \beta_{1-42}$ and CSF p-tau ${ }_{181 \mathrm{p}}$ levels (but not CSF p-tau ${ }_{181 \mathrm{p}}$ levels alone, Fig. 3 B2) were included as a covariate (Fig. 3B1 and 3B3).

\section{$A P O E$-by-sex interaction on brain structure}

Fig. 4A presents the vertex-wise interaction analysis across the whole cortical mantle, covaried by age and years of education, showing voxels with 

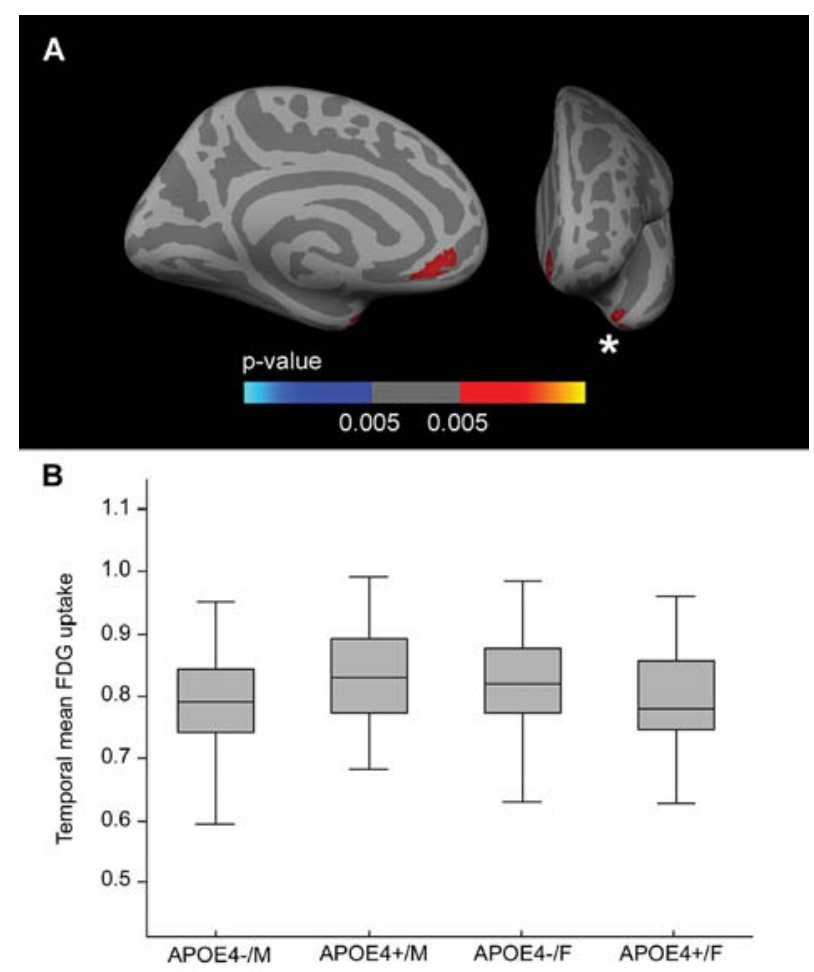

Figure 1: FDG $\boldsymbol{A P O E}$-by-sex interaction analysis. A. Areas in which there is a FDG-uptake interaction between sex and the APOE4 status ( $p<0.005$ uncorrected) co-varied for age and years of education displayed across the medial and frontal views of the cerebral cortex. B. Box and whisker plot illustrating individual FDG-uptake values in the temporal cluster. For each plot, the central black lines show the median value, the regions above and below the black line show the upper and lower quartiles, respectively, and the whiskers extend to the minimum and maximum values. As illustrated, the female APOE4 carriers showed decreased metabolism in the temporal cortex with respect to female non-carriers. $\mathrm{FDG}=$ fluorodeoxyglucose; $A P O E=$ apolipoprotein $\mathrm{E}, A P O E 4+=$ apolipoprotein $\mathrm{E} \varepsilon 4$ allele carriers, APOE4- = apolipoprotein E $\varepsilon 4$ allele non-carriers.

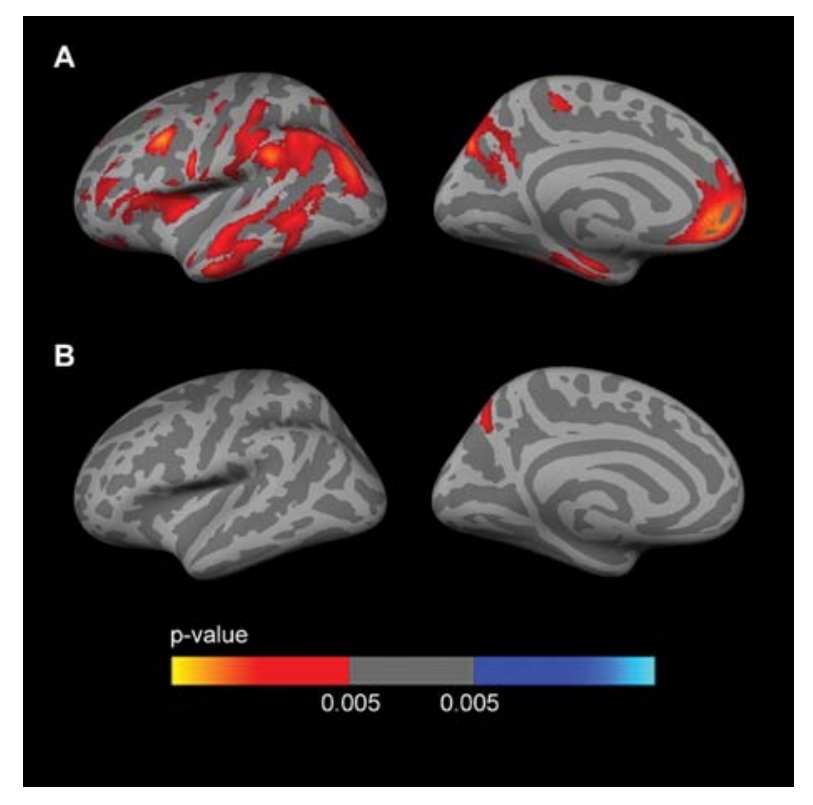

Figure 2: Sex-stratified FDG analyses. Analysis between APOE4 carriers and APOE4 non-carriers $(p<0.005$ uncorrected) in A. females and B. males, co-varied for age and years of education across the lateral and medial views of the cerebral cortex. As shown, female APOE4 carriers showed widespread clusters of decreased metabolism with respect to female APOE4 non-carriers (Fig. 2A), whereas male $A P O E 4$ carriers only showed an isolated cluster of decreased metabolism $(p<0.005)$ in the precuneus with respect to male non-carriers (Fig. 2B). FDG = fluorodeoxyglucose; $A P O E 4=$ apolipoprotein $\mathrm{E} \varepsilon 4$ allele. 


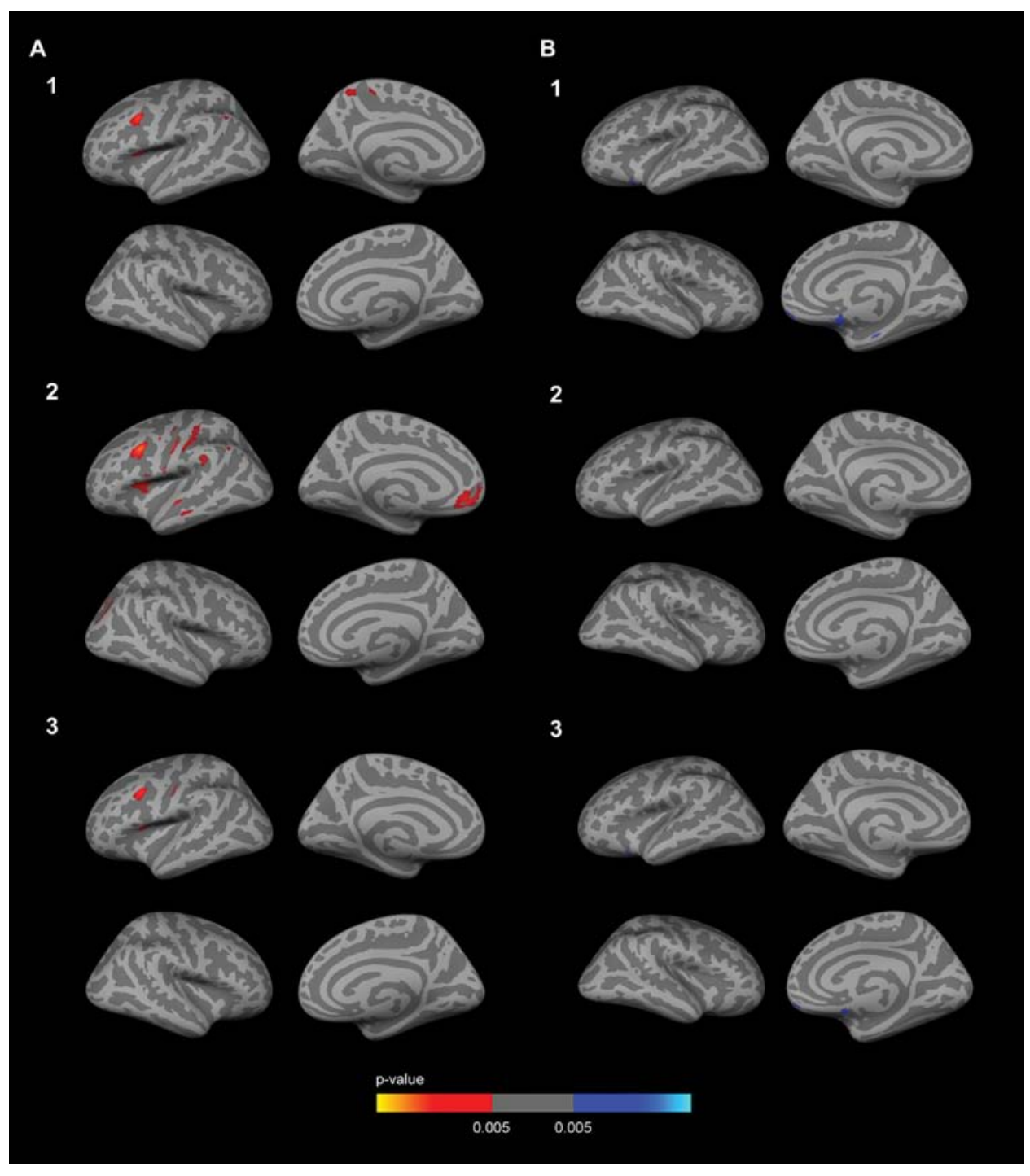

Figure 3: Sex-stratified FDG analyses with CSF biomarker levels included as a covariate. Row 1 . CSF A $\beta_{1-42}$ levels; Row 2. CSF p-tau ${ }_{181 \mathrm{p}}$ levels; Row 3 CSF A $\beta_{1-42}$ and p-tau ${ }_{181 \mathrm{p}}$ levels. The analysis between female $A P O E 4$ carriers and female $A P O E 4$ non-carriers A1-A3. showed several clusters of decreased metabolism ( $p<0.005$ uncorrected) co-varied for age. As illustrated, female $A P O E 4$ carriers showed decreased metabolism in the anterior cingulate cortex with respect to female non-carriers after the inclusion of the CSF biomarkers as a covariate. The analysis between male $A P O E 4$ carriers and male APOE4 non-carriers B1-B3. showed several clusters of increased metabolism ( $p<0.005$ uncorrected) co-varied for age. As illustrated, male APOE4 carriers showed increased metabolism in several clusters in the dorsolateral prefrontal cortex with respect to male $A P O E 4$ non-carriers after the inclusion of CSF $\mathrm{A} \beta_{1-42}$ levels or both CSF $\mathrm{A} \beta_{1-42}$ and CSF p-tau ${ }_{181 \mathrm{p}}$ as a covariate (B1 and B3), but not after the inclusion of the CSF p-tau ${ }_{181 \mathrm{p}}$ levels alone (B2). FDG $=$ fluorodeoxyglucose; $A P O E=$ apolipoprotein E, $A P O E 4$ : apolipoprotein $\mathrm{E} \varepsilon 4$ allele

an $A P O E$-by-sex interaction. Two large clusters (Family-wise error corrected $[\mathrm{FWE}] p<0.05$ ) emerged, one in the dorsolateral frontal region and one in the temporoparietal region. To analyze the directionality, we then isolated the temporoparietal cluster, averaged the cortical thickness (CTh), and plotted it in a box and whisker plot (Fig. 4B). As shown, this interaction was mainly driven by the increased $\mathrm{CTh}$ in male $A P O E 4$ carriers. The main effects and the interactive effects of $A P O E 4$ status and sex in the ANCOVA analysis were significant in the model (interaction term between $A P O E 4$ status and sex: $\beta$-coefficient $=-0.228, \mathrm{SE}=0.045$, $p<0.001$; main effect of sex: $\beta$-coefficient $=0.149$, $\mathrm{SE}=0.039, p<0.001$; main effect of APOE4 status: $\beta$-coefficient $=0.062, \mathrm{SE}=0.030, p=0.041$ ). Similar results were found for the remaining cluster (not shown).

Fig. 5 shows the sex-stratified APOE4 CTh analyses, covaried by age and years of education. Male APOE4 carriers showed 3 large clusters (FWE corrected) of increased CTh with respect to non-carriers. Two of the clusters were observed in the left hemisphere, one in the dorsolateral frontal region and another in the 

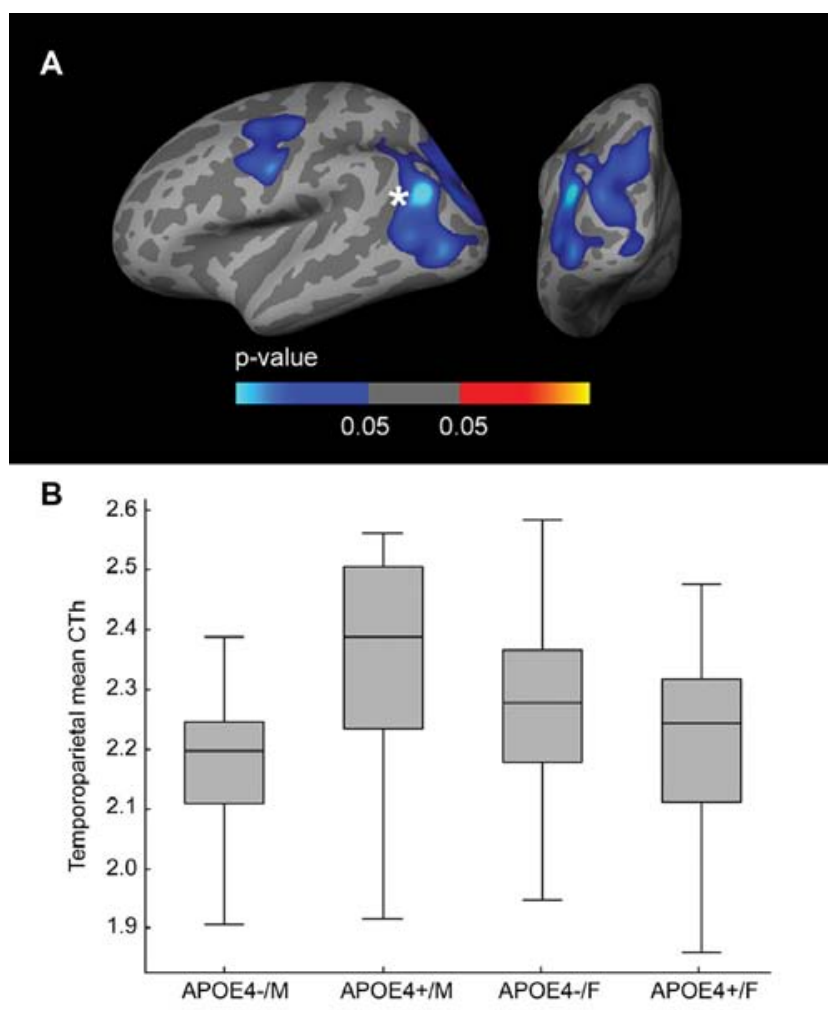

Figure 4: CTh $\boldsymbol{A P O E}$-by-Sex interaction analysis. A. Family-wise corrected $(p<0.05)$ clusters with an interaction between sex and the dichotomized $A P O E 4$ genotype co-varied for age and years of education displayed across the lateral and posterior views of the cerebral cortex. B. Box and whisker plot illustrating individual CTh values in the temporo-parietal and occipital cluster. For each plot, the central black lines show the median value, regions above and below the black line show the upper and lower quartiles, respectively, and the whiskers extend to the minimum and maximum values. As illustrated, male APOE4 carriers showed increased CTh in the temporo-parietal and occipital cluster. $\mathrm{CTh}=$ cortical thickness; $A P O E=$ apolipoprotein $\mathrm{E}, A P O E 4+=$ apolipoprotein $\mathrm{E} \varepsilon 4$ allele carriers, APOE4- = apolipoprotein E $\varepsilon 4$ allele non-carriers.

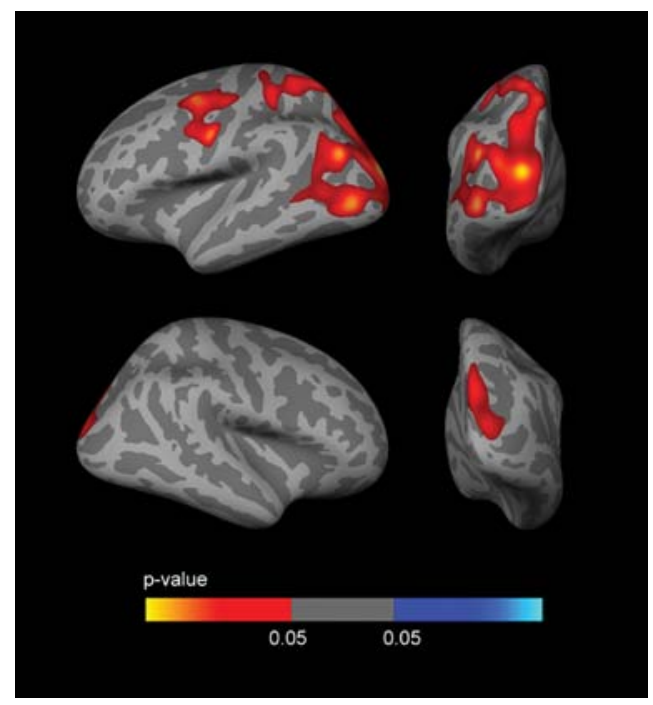

Figure 5: Sex-stratified CTh analyses. Analysis between male $A P O E 4$ carriers and male APOE4 non-carriers, co-varied for age and years of education. As shown, male APOE4 carriers presented large clusters of increased CTh (FWE $p<0.05)$ in temporo-parieto-occipital regions, mainly in the left hemisphere. The analysis between female APOE4 carriers and female APOE4 non-carriers showed clusters of decreased $\mathrm{CTh}$ which did not survive FWE correction (not shown). $\mathrm{CTh}=$ cortical thickness; $A P O E=$ apolipoprotein $\mathrm{E}$; FWE $=$ familywise error corrected $(p<0.05)$. 
temporoparietal, occipital and precuneus regions. The third cluster was observed in the right hemisphere in the parietal and occipital regions. Female APOE4 carriers showed cortical thinning in several regions than female APOE4 non-carriers (not shown as this analysis did not survive FWE correction).

To examine the influence of CSF biomarkers on the $A P O E$-by-sex interaction on brain structure, we included CSF $A \beta_{1-42}$ and CSF p-tau ${ }_{181 \mathrm{p}}$ as covariates in the analyses. The vertex-wise $A P O E$-by-sex interaction analysis across the whole cortical mantle showed a reduction in the significance maps when including CSF biomarkers as covariates, especially $\mathrm{A} \beta_{1-42}$ (Fig. 6). In the sex-stratified APOE4 CTh analyses, the clusters of increased $\mathrm{CTh}$ in male $A P O E 4$ carriers disappeared when CSF $A \beta_{1-42}$ levels (but not CSF p-tau ${ }_{181 p}$ levels) were included as a covariate (Fig. 7). No result survived FWE correction in females.

All analyses were repeated excluding APOE $\varepsilon 2$ allele carriers and including CSF t-tau as a covariate. We also restricted the analyses to non-hispanic white subjects (not shown). The results were not significantly altered in any case.

\section{DISCUSSION}

This study shows for the first time that the impact of the APOE4 genotype on brain structure and metabolism is modified by sex. We found a significant $A P O E$-by-sex interaction on brain metabolism and structure. Female APOE4 carriers showed brain hypometabolism and cortical thinning with respect to female non-carriers whereas male APOE4 carriers showed only a small cluster of hypometabolism and cortical thickening with respect to male non-carriers. CSF core AD biomarkers had an influence on brain structural results (and to a lesser extent on brain metabolism).

Epidemiologically, there is strong evidence that supports the $A P O E$-by-sex interaction [6], [11], [23]. The only study assessing the APOE-by-sex interactions on MRI demonstrated the interaction on resting state functional connectivity but not on gray matter volume [25]. Our results expand these findings. We show an $A P O E$-by-sex interaction on both brain structure and metabolism. The discrepancy on brain structure could be due to the differences in the subject population or technical differences (CTh analyses vs voxel-based morphometry [26]). Our FDG results are congruent with those of the aforementioned resting state functional connectivity analyses. $A P O E$ appears to affect brain network activity which is closely related to neuroenergetic functions [27].

Our metabolic findings suggest that women are metabolically more susceptible to the APOE4 genotype. Neglecting a possible $A P O E$-by-sex interaction on brain metabolism could be one of the reasons for the discordant FDG results [8]-[13]. Male APOE4 carriers showed increased CTh and females decreased CTh. The finding of cortical thickening in AD vulnerable areas in middle aged (48-75 years old) APOE4 carriers with respect to non-carriers has already been described [16], [17], but it is in contrast with other works assessing older cohorts [3], [4], [14], [15].

The discrepancies on brain structure might be conciliated if we consider a 2-phase phenomenon model in preclinical $\mathrm{AD}$ [28]. In this framework, pathological cortical thickening associated with low CSF A $\beta_{1-42}$ would be followed by atrophy once CSF p-tau ${ }_{181 \mathrm{p}}$ becomes abnormal [28]. Accordingly, our study shows that the clusters of increased $\mathrm{CTh}$ in male APOE4 carriers disappear when we included CSF $\mathrm{A} \beta_{1-42}$ as a covariate. The hypometabolism in female APOE4 carriers did not disappear when CSF $A \beta_{1-42}$ levels were included as a covariate. The $A P O E 4$ genotype might therefore exert its effects on brain glucose metabolism - at least in partindependently of amyloidogenic pathways [29]. Of note, the inclusion of CSF A $\beta_{1-42}$ levels as a covariate prompted the emergence of several areas of increased metabolism in male APOE4 carriers. Increased brain metabolism in relation to brain amyloidosis has been previously described [30].

Altogether, our findings support that the mechanisms underlying the increased AD risk in female $A P O E 4$ carriers might occur downstream of $A B$ pathology [6]. The APOE4 effect on lowering CSF A $\beta_{1-42}$ levels is marked in both men and women (with no sex differences) and was also found in our work [6], [25]. The impact of an $A P O E$-by-sex interaction on CSF has only been assessed twice and, as in the present work, always with data from the ADNI study. The absence of an $A P O E$ by-sex interaction on CSF $A \beta_{1-42}$ levels is in agreement with the two previous works [6], [25]. The impact on CSF p-tau ${ }_{181 \mathrm{p}}$ levels is less clear. We did not find an APOEby-sex interaction on CSF p-tau ${ }_{181 p}$ levels. Such an interaction was reported initially [25] in $\mathrm{HC}$ but was not confirmed in the later work with a larger sample size [6]. Nonetheless, this last work did find the interaction for CSF p-tau ${ }_{181 p}$ levels in mild cognitive impairment patients. Women, moreover, would be more susceptible and would present more abnormal neuronal injury biomarkers [25] and faster clinical decline [6]. Accordingly, female APOE4 carriers showed hypometabolism and cortical thinning with respect to non-carriers, suggesting that female APOE4 carriers might be more advanced in the aforementioned 2-phase phenomenon model in preclinical AD [28].

The mechanisms by which the $A P O E$ allele modifies the risk for $\mathrm{AD}$ have been extensively studied but are not completely understood. Both ß-amyloid-dependent [20] and $\beta$-amyloid-independent [21] mechanisms have been described. $A P O E$ appears to affect brain network activity and neuroenergetic functions [27] and to increase microglia reactivity at $A \beta$ plaques in mouse 


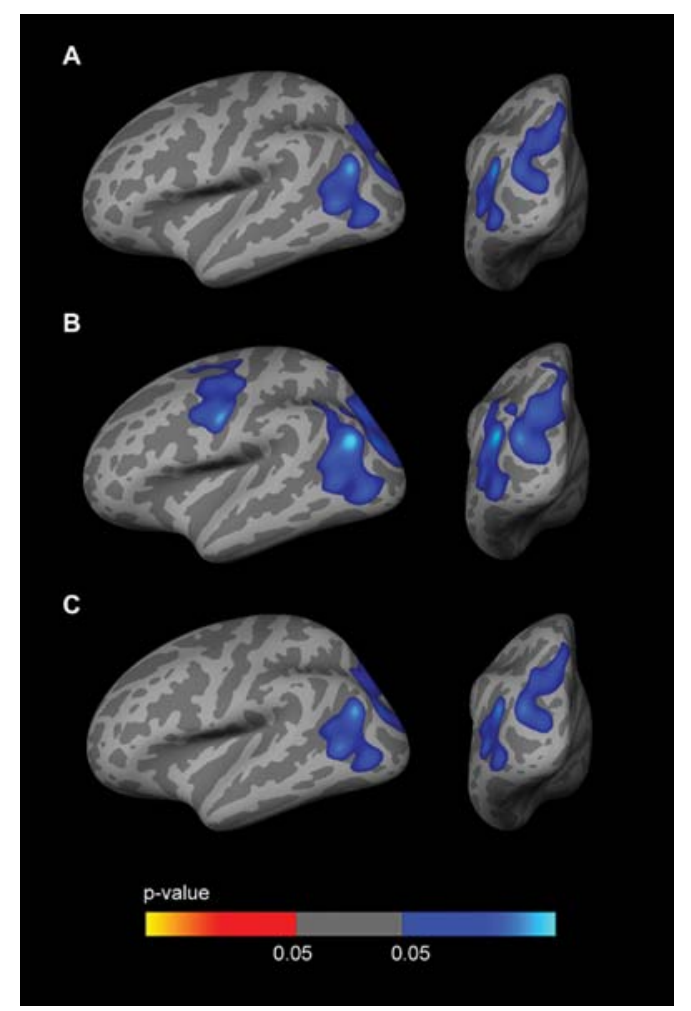

Figure 6: CTh APOE-by-Sex interaction analysis with CSF biomarker levels included as covariates. Family-wise corrected $(p<0.05)$ clusters with an interaction between sex and the dichotomized APOE4 genotype co-varied for age and: A. CSF A $\beta_{1-42}$ levels; B. CSF p-tau ${ }_{181 \mathrm{p}}$ levels; $\mathbf{C}$. CSF $A \beta_{1-42}$ and p-tau ${ }_{181 \mathrm{p}}$ levels. As illustrated, the inclusion of CSF $A \beta_{1-42}$ levels as a covariate significantly diminished the clusters showing a CTh $A P O E$-by-sex interaction. $\mathrm{CTh}=$ cortical thickness; $A P O E=$ apolipoprotein $\mathrm{E}$.

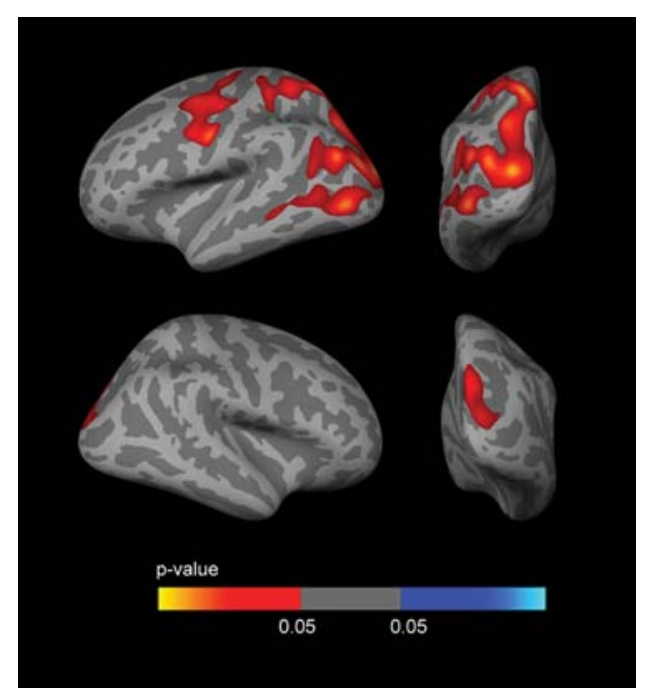

Figure 7: Sex stratified CTh analyses with CSF biomarker levels included as a covariate. The analysis between male APOE4 carriers and male APOE4 non-carriers showed several clusters of increased CTh $(p<0.005$ uncorrected) co-varied for age and CSF p-tau ${ }_{181 \mathrm{p}}$ levels. There were no significant clusters of increased CTh male APOE4 carriers vs male APOE4 non-carriers after the inclusion of $\mathrm{CSF} A \beta_{1-42}$ levels as a covariate. $\mathrm{CTh}=$ cortical thickness; $A P O E=$ apolipoprotein $\mathrm{E}$. 
models [31], [32]. These metabolic and inflammatory responses in relation to the $A P O E$ genotype might differ in males and females, accounting for the differences found.

This work has potential clinical implications. Clinical trials in preclinical AD in which APOE4 status is a selection criterion are underway (Alzheimer's Prevention Initiative APOE4 Trial, NIH project number 1UF1AG046150-01). Our results emphasize the importance of sex stratification when considering the $\mathrm{AD}$ risk and its impact on AD topographical biomarkers [33] conferred by the $A P O E$ genotype. More broadly, the present work stresses the need to consider interactions between biomarkers and risk factors in the $\mathrm{AD}$ preclinical phase [28].

The strengths of this study are the inclusion of a relatively high number of subjects and the fact that the results were found in two different topographical $A D$ biomarkers, [34] with congruent findings between the two. The study has some limitations. It is cross-sectional and the age-range sampled does not include young $\mathrm{HC}$ to assess the age-range in which amyloid is starting to deposit in the brain of APOE4 carriers [35].

In conclusion, the impact of APOE4 on brain structure and metabolism is modified by sex in HC. This interaction should be considered in current clinical trials in preclinical $\mathrm{AD}$ in which $A P O E 4$ status is a selection criterion.

\section{MATERIALS AND METHODS}

\section{Study participants and clinical classification}

Data used in the preparation of this article were obtained from the Alzheimer's Disease Neuroimaging Initiative (ADNI) database (http://adni.loni.usc.edu). The ADNI was launched in 2003 by the National Institute on Aging (NIA), the National Institute of Biomedical Imaging and Bioengineering (NIBIB), the Food and Drug Administration (FDA), private pharmaceutical companies and non-profit organizations, as a $\$ 60$ million, 5-year public-private partnership. The primary goal of ADNI has been to test whether serial magnetic resonance imaging (MRI), positron emission tomography (PET), other biological markers, and clinical and neuropsychological assessment can be combined to measure the progression of mild cognitive impairment (MCI) and early AD. Determination of sensitive and specific markers of very early AD progression is intended to aid researchers and clinicians to develop new treatments and monitor their effectiveness, as well as lessen the time and cost of clinical trials.

The Principal Investigator of this initiative is Michael W. Weiner, MD, VA Medical Center and University of California - San Francisco. ADNI is the result of efforts of many co-investigators from a broad range of academic institutions and private corporations, and subjects have been recruited from over 50 sites across the U.S. and Canada. The initial goal of ADNI was to recruit 800 subjects but ADNI has been followed by ADNI-GO and ADNI-2. To date these three protocols have recruited over 1500 adults, ages 55 to 90 , to participate in the research, consisting of cognitively normal older individuals (HC), people with early or late MCI, and people with early $\mathrm{AD}$. The follow up duration of each group is specified in the protocols for ADNI-1, ADNI-2 and ADNI-GO. Subjects originally recruited for ADNI-1 and ADNI-GO had the option to be followed in ADNI-2. For up-to-date information, see http://www.adni-info.org.

We included all $\mathrm{HC}$ with available CSF and/or a 3T-MRI and/or an FDG PET.

\section{CSF analyses}

\section{ADNI procedure}

Methods for CSF acquisition and biomarker measurement using the ADNI cohort have been reported previously [36]. $\mathrm{A} \beta_{1-42}$, total tau (t-tau) and phospho-tau $\left(\mathrm{p}-\mathrm{tau}_{181 \mathrm{p}}\right)$ levels were measured using the multiplex xMAP Luminex platform (Luminex) with Innogenetics (INNOBIA AlzBio3) immunoassay kit-based reagents.

\section{MRI and FDG-PET imaging procedures}

\section{ADNI acquisition procedure}

The details of MRI and FDG-PET acquisition are available elsewhere (http://www.adni-info.org).

\section{FDG-PET processing procedure}

FDG-PET images were downloaded in the most processed format. They were intensity-scaled by the reference pons-vermis region [37], spatially normalized using SPM8 [http://www.fil.ion.ucl.ac.uk/spm/] to the Montreal Neurological Institute (MNI) PET template and spatially smoothed with a Gaussian kernel of full width at half-maximum (FWHM) of $8 \mathrm{~mm}$. All resulting images were visually inspected to check for possible registration errors. Voxel-wise results were displayed at $p<0.005$ (uncorrected) using an extent threshold $k=50$, and projected on an inflated single-subject cortical surface reconstruction.

\section{Cortical thickness processing procedure}

Cortical reconstruction of the structural images was performed with the FreeSurfer software package, version 5.1 (http://surfer.nmr.mgh.harvard.edu). The procedures have been fully described elsewhere [38]. Estimated surfaces were inspected to detect errors in the automatic segmentation procedure. Fifty-seven of the 225 N3 processed MRI analyzed were excluded because 
of segmentation errors and 168 were included in the analyses. A Gaussian kernel of $15 \mathrm{~mm}$ full-width at half maximum was applied. To avoid false positives, we tested Monte Carlo simulation with 10,000 repeats in Qdec (family-wise error [FWE], $p<0.05$ ). Only regions that survived FWE are presented in the figures.

\section{Statistical methods}

Group analyses were made using SPSS (SPSS Inc, Chicago, IL). Comparisons between groups were performed using the two-tailed Student $t$ test for continuous variables and a chi-square test for categorical variables.

The main objective of our work was to study the $A P O E$-by-sex interaction on brain metabolism and brain structure. Two approaches were used: interaction and sex-stratified analyses. We carried out an ANCOVA as implemented in SPM and FreeSurfer for the PET and MRI analyses, respectively, using the $A P O E$ genotype ( $A P O E 4$ carrier vs $A P O E 4$ non-carrier) and sex as binary categorical independent variables, and age and years of education as variables of no interest to assess the interaction.

To examine the impact of CSF biomarkers on the FDG PET and CTh analyses, we introduced CSF biomarkers as covariates in the analyses. All analyses were repeated excluding APOE2 carriers and restricting to only non-hispanic white subjects.

Clusters derived from the interaction analyses in FDG or CTh were isolated to analyze the directionality of the interactive effects for each variable within an ANCOVA model, using age as a covariate. Specifically, we used the following model for FDG-PET and MRI:

Mean cluster FDG uptake (or mean cluster CTh) $=\hat{\mathrm{a}}_{0}+\hat{\mathrm{a}}_{1} * \mathrm{SEX}+\hat{\mathrm{a}}_{2} * A P O E+\mathrm{a}_{3} *[\mathrm{SEX} * A P O E]+$ age

The same ANCOVA approach was used for the CSF analyses to test for an interactive effect of $A P O E$ genotype and sex in CSF biomarker levels.

\section{ACKNOWLEDGMENTS}

We thank Carolyn Newey for editorial assistance.

\section{FUNDING}

This work was supported by research grants from the Carlos III Institute of Health, Spain (grants PI11/02425 and PI14/01126 to Juan Fortea, grants PI10/1878 and PI13/01532 to Rafael Blesa and PI11/03035 to Alberto Lleó) and the CIBERNED program (Program 1, Alzheimer Disease to Alberto Lleó), partly funded by FEDER funds of the EU. This work has also been supported by a "Marató TV3" grant (531/U/2014 to Juan Fortea). The work of Frederic Sampedro is supported by the Spanish government FPU (Formación del Profesorado Universitario) doctoral grant (Grant No. AP2012-0400). This work was also supported by NIH-NIA grants to M.J. de Leon, AG022374, AG13616, and AG12101.

Data collection and sharing for this project was funded by the Alzheimer's Disease Neuroimaging Initiative (ADNI) (National Institutes of Health Grant U01 AG024904) and DOD ADNI (Department of Defense award number W81XWH-12-2-0012). ADNI is funded by the National Institute on Aging, the National Institute of Biomedical Imaging and Bioengineering, and through generous contributions from the following: Alzheimer's Association; Alzheimer's Drug Discovery Foundation; BioClinica, Inc.; Biogen Idec Inc.; Bristol-Myers Squibb Company; Eisai Inc.; Elan Pharmaceuticals, Inc.; Eli Lilly and Company; F. Hoffmann-La Roche Ltd and its affiliated company Genentech, Inc.; GE Healthcare; Innogenetics, N.V.; IXICO Ltd.; Janssen Alzheimer Immunotherapy Research \& Development, LLC.; Johnson \& Johnson Pharmaceutical Research \& Development LLC.; Medpace, Inc.; Merck \& Co., Inc.; Meso Scale Diagnostics, LLC.; NeuroRx Research; Novartis Pharmaceuticals Corporation; Pfizer Inc.; Piramal Imaging; Servier; Synarc Inc.; andTakeda Pharmaceutical Company. The Canadian Institutes of Health Research is providing funds tosupport ADNI clinical sites in Canada. Private sector contributions are facilitated by the Foundation for the National Institutes of Health (http:// www.fnih.org). The grantee organization is the Northern California Institute for Research and Education, and the study is coordinated by the Alzheimer's Disease Cooperative Study at the University of California, San Diego. ADNI data are disseminated by the Laboratory for Neuro Imaging at the University of Southern California.

\section{CONFLICTS OF INTEREST}

All authors report no biomedical financial interests or potential conflicts of interest related to this work.

\section{REFERENCES}

1. Corder EH, Saunders AM, Strittmatter WJ, Schmechel DE, Gaskell PC, Small GW, Roses AD, Haines JL, PericakVance MA. Gene dose of apolipoprotein E type 4 allele and the risk of Alzheimer,'s disease in late onset families. Science. 1993; Aug 261:921-3.

2. Liu C-C, Liu C-C, Kanekiyo T, Xu H, Bu G. Apolipoprotein $\mathrm{E}$ and Alzheimer disease: risk, mechanisms and therapy. Nat. Rev. Neurol. 2013; Feb 9:106-18.

3. Liu Y, Yu J-T, Wang H-F, Han P-R, Tan C-C, Wang C, Meng X-F, Risacher SL, Saykin AJ, Tan L. APOE genotype and neuroimaging markers of Alzheimer's disease: systematic review and meta-analysis. J. Neurol. Neurosurg. Psychiatry. 2015; May 86:127-34. 
4. Reinvang I, Espeseth T, Westlye LT. APOE-related biomarker profiles in non-pathological aging and early phases of Alzheimer's disease. Neurosci. Biobehav. Rev. 2013; May 37:1322-1335.

5. Sunderland T, Mirza N, Putnam KT, Linker G, Bhupali D, Durham R, Soares H, Kimmel L, Friedman D, Bergeson J, Csako G, Levy JA, Bartko JJ, Cohen RM. Cerebrospinal fluid beta-amyloid1-42 and tau in control subjects at risk for Alzheimer's disease: the effect of APOE epsilon4 allele. BiolPsychiatry. 2004; Nov 56:670-6.

6. Altmann A, Tian L, Henderson VW, Greicius MD. Sex modifies the APOE-related risk of developing Alzheimer disease. AnnNeurol. 2014; Apr 75:563-73.

7. Vemuri P, Wiste HJ, Weigand SD, Knopman DS, Shaw LM, Trojanowski JQ, Aisen PS, Weiner M, Petersen RC, Jack CR. Effect of apolipoprotein E on biomarkers of amyloid load and neuronal pathology in Alzheimer disease. AnnNeurol. 2010; Mar 67:308-16.

8. Reiman EM, Caselli RJ, Yun LS, Chen K, Bandy D, Minoshima S, Thibodeau SN, Osborne . Preclinical evidence of Alzheimer's disease in persons homozygous for the epsilon 4 allele for apolipoprotein E. N. Engl. J. Med. 1996; Mar 334:752-8.

9. Reiman EM, Chen K, Alexander GE, Caselli RJ, Bandy D, Osborne D, Saunders AM, Hardy J. Correlations between apolipoprotein E epsilon4 gene dose and brain-imaging measurements of regional hypometabolism. Proc. Natl. Acad. Sci. U. S. A. 2005; Jun 102:8299-302.

10. Reiman EM, Chen K, Alexander GE, Caselli RJ, Bandy D, Osborne D, Saunders AM, Hardy J. Functional brain abnormalities in young adults at genetic risk for late-onset Alzheimer's dementia. Proc. Natl. Acad. Sci. U. S. A. 2004; Jan 101:284-9.

11. Corder EH, Jelic V, Basun H, Lannfelt L, Valind S, Winblad B, Nordberg A. No difference in cerebral glucose metabolism in patients with Alzheimer disease and differing apolipoprotein E genotypes. ArchNeurol. 1997; Mar 54:273-7.

12. Hirono N, Mori E, Yasuda M, Imamura T, Shimomura T, Hashimoto M, Tanimukai S, Kazui H, Yamashita H. Lack of effect of apolipoprotein E E4 allele on neuropsychiatric manifestations in Alzheimer's disease. J. Neuropsychiatry Clin. Neurosci. 1999; Jan 11:66-70.

13. Samuraki M, Matsunari I, Chen W-P, Shima K, Yanase D, Takeda N, Matsuda H, Yamada M. Glucose metabolism and gray-matter concentration in apolipoprotein $\mathrm{E} \varepsilon 4$ positive normal subjects. NeurobiolAging. 2012; Oct 33:2321-3.

14. Cherbuin N, Leach LS, Christensen H, Anstey KJ. Neuroimaging and APOE genotype: a systematic qualitative review. Dement. Geriatr. Cogn. Disord. 2007; Jan 24:348-62.

15. Novak NM, Stein JL, Medland SE, Hibar DP, Thompson PM, Toga AW. EnigmaVis: online interactive visualization of genome-wide association studies of the Enhancing
NeuroImaging Genetics through Meta-Analysis (ENIGMA) consortium. Twin Res. Hum. Genet. 2012; Jun 15:414-8.

16. Espeseth T, Westlye LT, Fjell AM, Walhovd KB, Rootwelt H, Reinvang I. Accelerated age-related cortical thinning in healthy carriers of apolipoprotein E epsilon 4. NeurobiolAging. 2008; Mar 29:329-340.

17. Espeseth T, Westlye LTL, Walhovd KKB, Fjell AM, Endestad T, Rootwelt H, Reinvang I. Apolipoprotein E \&4-related thickening of the cerebral cortex modulates selective attention. NeurobiolAging. 2012; Mar 33:304-322.e1.

18. Filippini N, Ebmeier KP, MacIntosh BJ, Trachtenberg AJ, Frisoni GB, Wilcock GK, Beckmann CF, Smith SM, Matthews PM, Mackay CE. Differential effects of the APOE genotype on brain function across the lifespan. Neuroimage. 2011; Jan 54:602-10.

19. Dean DC, Jerskey BA, Chen K, Protas H, Thiyyagura P, Roontiva A, O'Muircheartaigh J, Dirks H, Waskiewicz N, Lehman K, Siniard AL, Turk MN, Hua X, Madsen SK, Thompson PM, Fleisher AS, Huentelman MJ, Deoni SCL, Reiman EM. Brain differences in infants at differential genetic risk for late-onset Alzheimer disease: a cross-sectional imaging study. JAMA Neurol. 2014; Jan 71:11-22.

20. Holtzman DM, Herz J, Bu G. Apolipoprotein E and apolipoprotein $\mathrm{E}$ receptors: normal biology and roles in Alzheimer disease. Cold Spring Harb. Perspect. Med. 2012; Mar 2:a006312.

21. Wolf AB, Valla J, Bu G, Kim J, LaDu MJ, Reiman EM, Caselli RJ. Apolipoprotein E as a $\beta$-amyloid-independent factor in Alzheimer's disease. Alzheimers. Res. Ther. 2013; Jan 5:38.

22. Poirier J, Davignon J, Bouthillier D, Kogan S, Bertrand P, Gauthier S. Apolipoprotein E polymorphism and Alzheimer's disease. Lancet. 1993; Sep 342:697-9.

23. Payami H, Montee KR, Kaye JA, Bird TD, Yu CE, Wijsman EM, Schellenberg GD. Alzheimer's disease, apolipoprotein E4, and gender. JAMA. 1994; May 271:1316-7.

24. Farrer LA, Cupples LA, Haines JL, Hyman B, Kukull WA, Mayeux R, Myers RH, Pericak-Vance MA, Risch N, van Duijn CM. Effects of age, sex, and ethnicity on the association between apolipoprotein E genotype and Alzheimer disease. A meta-analysis. APOE and Alzheimer Disease Meta Analysis Consortium. JAMA. 278:1349-561997.

25. Damoiseaux JS, Seeley WW, Zhou J, Shirer WR, Coppola G, Karydas A, Rosen HJ, Miller BL, Kramer JH, Greicius MD. Gender modulates the APOE $\varepsilon 4$ effect in healthy older adults: convergent evidence from functional brain connectivity and spinal fluid tau levels. JNeurosci. 2012; Jun 32:8254-62.

26. Fortea J, Sala-Llonch R, Bartrés-Faz D, Bosch B, Lladó A, Bargalló N, Molinuevo JL, Sánchez-Valle R. Increased cortical thickness and caudate volume precede atrophy in PSEN1 mutation carriers. J. Alzheimers. Dis. 2010; Jan 22:909-22. 
27. Wolf AB, Caselli RJ, Reiman EM, Valla J. APOE and neuroenergetics: an emerging paradigm in Alzheimer's disease. NeurobiolAging. 2013; Apr 34:1007-17.

28. Fortea J, Vilaplana E, Alcolea D, Carmona-Iragui M, Sánchez-Saudinos M-B, Sala I, Antón-Aguirre S, González S, Medrano S, Pegueroles J, Morenas E, Clarimón J, Blesa R, Lleó A. Cerebrospinal Fluid B-Amyloid and Phospho-Tau Biomarker Interactions Affecting Brain Structure in Preclinical Alzheimer Disease. AnnNeurol. 2014; May :1-8.

29. Jagust WJ, Landau SM. Apolipoprotein E, not fibrillar $\beta$-amyloid, reduces cerebral glucose metabolism in normal aging. JNeurosci. 2012; Dec 32:18227-33.

30. Johnson SC, Christian BT, Okonkwo OC, Oh JM, Harding S, Xu G, Hillmer AT, Wooten DW, Murali D, Barnhart TE, Hall LT, Racine AM, Klunk WE, a Mathis C, Bendlin BB, Gallagher CL, Carlsson CM, a Rowley H, Hermann BP, Dowling NM, Asthana S, a Sager M. Amyloid burden and neural function in people at risk for Alzheimer's Disease. NeurobiolAging. 2014; Mar 35:576-84.

31. Rodriguez GA, Tai LM, LaDu MJ, Rebeck GW. Human APOE4 increases microglia reactivity at $A \beta$ plaques in a mouse model of $A \beta$ deposition. JNeuroinflammation. 2014; Jan 11:111.

32. Tai LM, Ghura S, Koster KP, Liakaite V, MaienscheinCline M, Kanabar P, Collins N, Ben-Aissa M, Lei AZ, Bahroos N, Green S, Hendrickson B, Van Eldik LJ, LaDu MJ. APOE -modulated Aß-induced neuroinflammation in Alzheimer's disease: current landscape, novel data and future perspective. JNeurochem. 2015; Feb.
33. Sperling R, Aisen P, Beckett L. Toward defining the preclinical stages of Alzheimer's disease: Recommendations from the National Institute on Aging-Alzheimer's Association workgroups workgroups on diagnostic guidelines for Alzheimer's disease. 2011; May 7:280-92.

34. Dubois B, Feldman HH, Jacova C, Cummings JL, Dekosky ST, Barberger-Gateau P, Delacourte A, Frisoni G, Fox NC, Galasko D, Gauthier S, Hampel H, Jicha GA, Meguro K, O'Brien J, Pasquier F, Robert P, Rossor M, Salloway S, Sarazin M, de Souza LC, Stern Y, Visser PJ, Scheltens P. Revising the definition of Alzheimer's disease: a new lexicon. Lancet Neurol. 2010; Nov 9:1118-27.

35. Pletnikova O, Rudow G, Hyde T, Kleinman JE, Ali S, Bharadwaj R, Gangadeen S, Crain B, Fowler D, Rubio A, Troncoso J. Alzheimer lesions in the brains of young subjects. Cogn. Behav. Neurol. 2015; In press.

36. Shaw LM, Vanderstichele H, Knapik-Czajka M, Clark CM, Aisen PS, Petersen RC, Blennow K, Soares H, Simon A, Lewczuk P, Dean R, Siemers E, Potter W, Lee V M-Y, Trojanowski JQ, Initiative ADN. Cerebrospinal Fluid Biomarker Signature in Alzheimer's Disease Neuroimaging Initiative Subjects. AnnNeurol. 2009; Apr 65:403-413.

37. Landau S, Jagust W. UC Berkeley FDG MetaROI methods. Alzheimer's Dis. Neuroimaging Initiat. 2011.

38. Fischl B, Dale AM. Measuring the thickness of the human cerebral cortex from magnetic resonance images. Proc. Natl. Acad. Sci. U. S. A. 2000; Sep 97:11050-5. 